\title{
Phase Contrast Cone Beam Tomography with an X-Ray Grating Interferometer
}

\author{
I. Jerjen ${ }^{\mathrm{a}}$, V. Revol ${ }^{\mathrm{b}, \mathrm{c}}$, C. Kottler ${ }^{\mathrm{b}}$, Th. Luethi ${ }^{\mathrm{a}}$, U. Sennhauser ${ }^{\mathrm{a}}$, R. Kaufmann ${ }^{\mathrm{b}}$, and \\ C. Urban ${ }^{\mathrm{b}}$ \\ ${ }^{a}$ Laboratory for Electronics/Metrology/Reliability, Empa, Ueberlandstrasse 129, CH-8600 Duebendorf, Switzerland \\ ${ }^{b}$ Photonics Division, CSEM SA, Technoparkstrasse 1, CH-8005 Zuerich, Switzerland \\ ${ }^{c}$ Physics Institute, University of Zuerich, Winterthurerstrasse 190, CH-8057 Zuerich, Switzerland
}

\begin{abstract}
We report on our recent developments of reconstruction algorithms for Differential Phase Contrast X-ray Computed Tomography (DPC CT). DPC images provide information about the real and imaginary part of the refractive index which is an advantage when objects with poor absorption but good phase contrast are inspected. In order to promote DPC CT for industrial applications we developed an adapted Feldkamp algorithm which allows reconstructing the three-dimensional image of the refractive index of an object from the DPC projections obtained with our large field of view, high energy grating interferometer set up in a cone beam geometry. We present slice images of a test object and show different ways of visualization of the phase and absorption information.
\end{abstract}

Keywords: X-ray, Computed Tomography, Differential Phase Contrast, Cone Beam, Feldkamp Algorithm PACS: 42.30.Rx, 42.30.Wb, 41.50.+h, 07.85.-m

\section{INTRODUCTION}

X-ray computed tomography (CT) is a well-established tool for obtaining three-dimensional images of objects and is well-established in life science and in industry. Traditionally a series of transmission images of a test sample measured at different angles is recorded and a three-dimensional representation of the sample is calculated by numerical methods, prevalently by applying a Filtered Back-Projection (FBP) algorithm ${ }^{1,2}$. However CT based on the absorption of X-rays reaches its limits if the object under test exhibits weak absorption contrast, either between the object itself and the ambient medium (most commonly air or water) or between different parts of the object. Yet in certain cases the difference of the phase shift is important enough to result in a phase contrast which exceeds the absorption contrast $\mathrm{t}^{3,14}$. Therein is the interest to develop methods for X-ray phase contrast CT. However, from the various phase retrieval techniques $^{3-14}$ only propagation $^{9-11}$ and grating ${ }^{12-14}$ based methods are appropriate to be used with cheap X-ray tubes which have a broad energy spectrum ${ }^{17}$.

In traditional absorption contrast X-ray CT a cone beam set up is often used either in order to get a magnified image of the investigated object $(\mu \mathrm{CT})$ or in order to avoid scanning the object and therefore reducing the measurement time. The reconstruction of the three-dimensional image is conventionally done by applying a Feldkamp algorithm ${ }^{1,2}$. A cone beam setup is also possible for phase contrast X-ray CT as was demonstrated by several research groups ${ }^{9,17}$.

We applied a source grating in order to transform an ordinary X-ray tube into a locally coherent X-ray source and used a Talbot interferometer set up to obtain simultaneously Differential Phase Contrast (DPC), Absorption Contrast (AC) and Dark Field (DF) images of a test object. Since our set up corresponds to a cone beam setup with a small opening angle we employed a modified Feldkamp algorithm for the reconstructions of the three-dimensional distribution of the attenuation coefficient $\mu$ and the refractive index $\delta$. The three-dimensional images are displayed separately and also visualized in a composite image combining the phase and absorption information. 


\section{EXPERIMENT}

We recorded the phase and absorption contrast images of a "Surprise Egg" at 720 angles between 0 and 360 degrees, combining a conventional cone beam X-ray CT setup with a Talbot-Lau interferometer. The "Surprise Egg" is made of some plastic toys inside a plastic container which is within a hull made of chocolate. The whole "Surprise Egg" is wrapped into an Al foil (see Figure $1 \mathrm{f}$ ).

A x-ray tube from COMET (MXR-160HP 20) with focal spot size of $1 \times 1 \mathrm{~mm}^{2}$ and operated at an acceleration voltage of $40 \mathrm{kV}$, a flat-panel x-ray detector from Radicon (Shad-o-Box 2048, scintillator Min-R 2190) with 2048 $\times$ 1024 pixels of size $48 \times 48 \mu \mathrm{m}^{2}$ and a precision rotation stage from Owis (DMT 65) constitute the conventional CT part of the setup.

We obtained DPC images by means of a Talbot-Lau X-ray interferometer setup ${ }^{15}$ consisting of three gratings which were fabricated in the clean room of the CSEM in Neuchâtel by a process combining lithography, deep reactive ion etching, wet etching and gold electroplating. The source grating $\mathrm{G}_{0}$ with a periodicity of $57 \mu \mathrm{m}$ and a depth of $60 \mu \mathrm{m}$ generates individually coherent light sources. The phase grating $\mathrm{G}_{1}$ with a periodicity of $2.85 \mu \mathrm{m}$ and a depth of $12.7 \mu \mathrm{m}$ imprints phase modulations on the incoming X-ray beam and thus creates interference fringes. The transmission grating $\mathrm{G}_{2}$ with a periodicity of $3 \mu \mathrm{m}$ and a depth of $30 \mu \mathrm{m}$ acts as an analyzer and allows scanning through the interference pattern. The dimensions of the grids have been optimized for an x-ray energy of about $20 \mathrm{keV}$.

We executed the phase stepping by moving the source grating $\mathrm{G}_{0}$ in 12 increments of $5 \mu \mathrm{m}$ ensuring a scan of the full fringe pattern period. In our setup the source grating $\mathrm{G}_{0}$ was mounted on the $\mathrm{x}$-ray tube and both parts were moved together employing a linear stage from Newport (UTS100PP) with mechanical uncertainty in the order of $0.3 \mu \mathrm{m}$.

The distances between the source grating $G_{0}$ and the phase grating $G_{1}$ was $1310 \mathrm{~mm}$ and the distance between the phase grating $G_{1}$ and the transmission grating $G_{2}$ was $69 \mathrm{~mm}$. The distance between the x-ray source and the test object was $1285 \mathrm{~mm}$ and the distance between the source and the detector was $1404 \mathrm{~mm}$.

\section{RECONSTRUCTION}

The intensity of one pixel $I_{i, j}$ as a function of the phase step $k$ can, for a polychromatic spectrum, be approximated by ${ }^{19,20}$ :

$$
I_{i j}(k) \approx A_{i j}\left[1+v_{i j} \sin \left(2 \pi \frac{k x_{0}}{L}+\varphi_{i j}\right)\right],
$$

where $\mathrm{A}_{\mathrm{ij}}$ is the mean intensity of a pixel, $\mathrm{v}_{\mathrm{ij}}$ a measure of the modulation strength due to the interference, $\mathrm{x}_{0}$ the step size, $\mathrm{L}$ the period of the interference pattern and $\varphi_{\mathrm{ij}}$ the phase of the sine curve. Provided that the phase stepping occurs over a whole period $L$, the values of $A_{i j}, v_{i j}$ and $\varphi_{i j}$ can be obtained from the measured values $I_{i, j}(k)$ with the help of the Discrete Fourier Transformation (DFT): The mean intensity is given by the real part of the DC component, the modulation strength and the phase are given by the modulus and the argument of the first frequency component respectively.

The mean intensity image $\mathrm{A}_{\mathrm{ij}}$ corresponds to the conventional absorption contrast image and therefore a standard Feldkamp Filtered Backprojection (FPB) algorithm with a Ram-Lak filter ${ }^{1,2}$ was used for the tomographic reconstruction of the 3-dimensional distribution of the absorption coefficient $\mu$.

The phase $\varphi$ of the interference pattern is a measure of the angular deflection $\alpha$ of the X-rays caused by a phase object placed in the X-ray beam path. The deflection angle is proportional to the local gradient of the object's phase shift $^{15-17}$ :

$$
\varphi=\alpha=\frac{\lambda}{2 \pi} \frac{\partial \Phi(y, z)}{\partial y}=\int_{-\infty}^{\infty} \frac{\partial \delta(x, y, z)}{\partial y} d x
$$

where $\lambda$ is the wavelength of the X-rays and $\delta(x, y, z)$ the decrement of the real part of the object's refractive index $\mathrm{n}(\mathrm{x}, \mathrm{y}, \mathrm{z})$. It was shown that $\delta(\mathrm{x}, \mathrm{y}, \mathrm{z})$ can be reconstructed directly from the differential phase contrast images $\varphi_{\mathrm{ij}}$ by replacing the standard FBP filter function ${ }^{15,16,18}$ : 


$$
\tilde{k}(v)= \begin{cases}|v|, & |v| \leq \frac{1}{2 \Delta y} \\ 0, & |v|>\frac{1}{2 \Delta y}\end{cases}
$$

by

$$
\tilde{h}(v)=\left\{\begin{array}{cc}
\frac{1}{2 \pi i} \operatorname{sign}(v), & |v| \leq \frac{1}{2 \Delta y}, \\
0, & |v|>\frac{1}{2 \Delta y}
\end{array},\right.
$$

where $\mathrm{v}$ is the reciprocal space coordinate corresponding to $\mathrm{x}$ and $\Delta \mathrm{y}$ is the sampling interval in the $\mathrm{y}$ direction. After this replacement the standard Feldkamp algorithm is used to obtain the 3-dimensional distribution of the real part of the object's refractive index.

By using the Feldkamp algorithm the reconstruction takes into account that the measurements were carried out in a cone beam setup where the X-rays leave the object at a slightly different yz position then where they entered. For a diameter of our test object of about $54 \mathrm{~mm}$ the resulting position drift amounts to about $0.28 \mathrm{~mm}$ or 6.4 pixels. Therefore a reconstruction algorithm assuming a parallel beam setup would result in a blurred three-dimensional image of the object.

\section{RESULTS}

The three-dimensional absorption and phase images, corresponding to the real and imaginary part of the refractive index, were reconstructed from the measured absorption and differential phase contrast projections as explained above. One representative slice of the test object is shown in Figure 1: For comparison purposes the intensity values of the images were scaled in such a way that one material, the chocolate, shows the same grey value in both images. The absorption image (see Figure 1 a) shows that the Al wrapping has the highest absorption of Xrays followed by the chocolate and the plastics. Parts of the plastic toy which show a more important absorption were identified as the coloring. The phase image (see Figure $1 \mathrm{~b}$ ) shows almost no difference of the real part of the refractive index between the chocolate and the plastics. The Al wrapping can't be seen at all. A closer inspection reveals that the position resolution of the phase image is slightly worse than the position resolution of the absorption image. Therefore the thin Al foil, approximately $20 \mu \mathrm{m}$ thick, can't be resolved. So in the case of our test object, the absorption image allows better differentiating of the materials than the phase image while in other cases the contrary could occur. This leads to the question how both images can be combined in order to have an optimum contrast between different materials. Here we propose to combine the real and imaginary part of the refractive index information into an RGB color image in such a way that the difference between the absorption and phase image is represented in two color channels. For this purpose we scaled the intensities of the absorption and phase images so that the difference of the scaled images shows negative and positive values (see Figure $1 \mathrm{c}$ ). Then we added the absolute values of the negative and positive parts to two copies of the reference image, which can be either the absorption or the phase image. The two resulting images constitute the red and green channels respectively and the blue channel contains the reference image. Depending on the reference image the plastic or the Al wrapping are pronounced a little bit more (see Figure $1 \mathrm{~d}$ with the absorption image as reference and Figure $1 \mathrm{e}$ with the phase image as reference). But in both cases the plastics, chocolate and $\mathrm{Al}$ foil are clearly separated from each other. The coloring of the plastic toy is clearly visible too. 

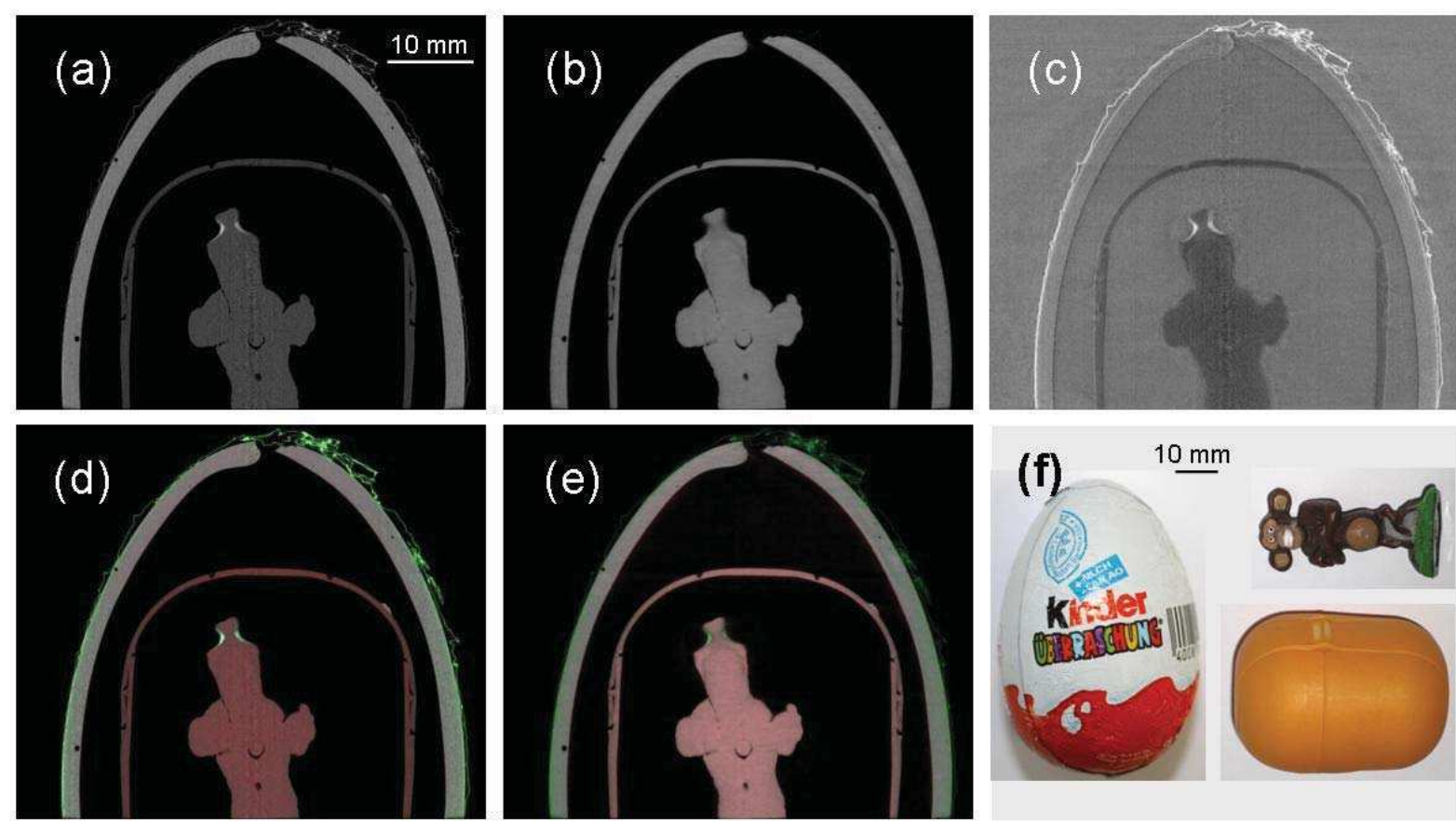

FIGURE 1. This figure shows the same slice of the reconstructed three-dimensional image of the test object, a "Surprise Egg", in different ways (pixels size $=44 \mu \mathrm{m}$ ): (a) is the absorption image, (b) the phase image, (c) the difference between the absorption and phase image. (d) and (e) are RGB color images obtained in slightly different ways: the red and green colors indicate regions

where the index of refraction $\delta$ or the attenuation coefficient $\mu$ are more pronounced respectively. (f) shows photos of the "Surprise Egg" and its contents.

\section{CONCLUSION}

We reconstructed the three-dimensional image of the refractive index distribution of a test object from the differential phase contrast projections which we obtained with the help of a Talbot-Lau interferometer set up in a cone beam configuration. We combined the absorption and phase information in a RGB color image in order to better visualize the test object. We could clearly separate the different materials which the test object is made of. However we couldn't retrieve any features or additional information from the combined measurement of the real and imaginary part of the test object which we couldn't see in the absorption image alone. In order to show which benefits Differential Phase Contrast Computed Tomography (DPC CT) offers in comparison to conventional absorption contrast CT one has to identify test objects with poor absorption contrast which are of practical interest, for example cancer tissue surrounded by healthy tissue or items made of different plastics. And then one has to compare the method to alternative methods as for example dual energy absorption contrast CT.

In order to implement DPC CT with micrometer resolution we have to quantify the observed resolution degradation of the DPC reconstruction and to investigate the reasons leading to this degradation. Also we have to employ grids which also accept strongly oblique X-rays. This will allow a cone beam setup with bigger opening angle necessary for a higher magnification.

\section{ACKNOWLEDGMENTS}

We gratefully acknowledge F. Cardot, P. Niedermann and the Division Microsystems Technology from CSEM in Neuchâtel for the gratings' fabrication.

This work was supported by the CCMX-NMMC platform (Grant Nr. 0206088). 


\section{REFERENCES}

1. A. C. Kak and M. Slaney, "Algorithms for Reconstruction with Non-diffracting Sources" in Principles of Computerized Tomographic Imaging, edited by R. F. Cotellessa, New York: IEEE Press (1988).

2. L. A. Feldkamp, L. C. Davis, and J. W. Kress, J. Op. Soc. Am., Vol. 1, 612 (1984).

3. R. Fitzgerald, Phys. Today 53(7), 23 (2000).

4. A. Momose, Opt. Express 11, 2303 (2003).

5. A. Momose, Jpn. J. Appl. Phys., Part 1 44, 6355 (2005).

6. U. Bonse, M. Hart, Appl. Phys. Lett., 6, 155 (1965).

7. A. Momose, T. Takeda, Y. Itai, and K. Hirano, Nature Medicine, 2, 473 (1996).

8. L. D. Chapman, W. C. Thomlinson, R. E. Johnston, D. Washburn, E. Pisano, N. Gmur, Z. Zhong, R. Menk, F. Arfelli, and D. Sayers, Phys. Med. Biol., 42, 2015 (1997).

9. S. W. Wilkins, T. E. Gureyev, D. Gao, A. Pogany, and A. W. Stevenson, Nature 384, 335 (1996).

10. G. R. Myers, S. C. Mayo, T. E. Gureyev, D. M. Paganin, and S. W. Wilkins, Phys. Rev. A 76, 45804 (2007).

11. P. Cloetens, W. Ludwig, J. Baruchel, D. van Dyck, J. van Landuyt, J. P. Guigay, M. Schlenker, Appl. Phys. Lett., 75, $2912(1999)$

12. C. David, B. Nöhammer, H. H. Solak, and E. Ziegler, Appl. Phys. Lett. 81, 3287 (2002).

13. F. Pfeiffer, T. Weitkamp, O. Bunk, and C. David, Nature Phys. 2, 258 (2006).

14. T. Weitkamp, A. Diaz, C. David, F. Pfeiffer, M. Stampanoni, P. Cloetens, and E. Ziegler, Opt. Express 13, 6396 (2005).

15. F. Pfeiffer, C. Kottler, O. Bunk, and C. David, Phys. Rev. Lett. 98, 108105 (2007).

16. F. Pfeiffer, C. Kottler, O. Bunk, and C. David, Nucl. Instrum. Methods Phys. Res. , A580, 925 (2007).

17. M. Engelhardt, J. Baumann, M. Schuster, C. Kottler, F. Pfeiffer, O. Bunk, and C. David, Appl. Phys. Lett. 90, 224101 (2007).

18. G. W. Faris, R. L. Byer, Appl. Opt. 27, 5202 (1988).

19. A. Momose, W. Yashiro, Y. Takeda, Y. Suzuki, and T. Hattori, Jpn. J. Appl. Phys., 45, 5254 (2006).

20. F. Pfeiffer, M. Bech, O. Bunk, P. Kraft, E. Eikenberry, C. Brönnimann, C. Grünzweig, and C. David, Nature Materials 7 , $134(2008)$ 